\title{
Characterising the Baseband Impedance of Supply Modulators Using Simple Modulated Signals
}

\author{
Alexander Alt \\ Cardiff University \\ Cardiff, UK \\ alta@cardiff.ac.uk
}

\author{
Andrew Forsyth \\ University of Manchester \\ Manchester, UK \\ andrew.forsyth@manchester.ac.uk
}

\author{
Alejandro Villarruel-Parra \\ University of Manchester \\ Manchester, UK \\ a.villarruel@manchester.ac.uk
}

\author{
Peng Chen \\ Cardiff University \\ Cardiff, UK \\ chenp10@ cardiff.ac.uk
}

\author{
Sattam Alsahali \\ Cardiff University \\ Cardiff, UK \\ alsahalisf@cardiff.ac.uk
}

\author{
Paul J. Tasker \\ Cardiff University \\ Cardiff, UK \\ tasker@cardiff.ac.uk
}

\author{
Jonathan Lees \\ Cardiff University \\ Cardiff, UK \\ leesj2@cardiff.ac.uk
}

\begin{abstract}
Envelope tracking is one of the promising technologies for $5 \mathrm{G}$ power amplifiers, providing high power efficiency over a wide output power range by modulating the supply voltage. Although the baseband impedance, the output impedance of the supply modulator, plays a crucial role in the linearity of the PA, it is often not measured or considered during the modulator design. This paper presents a new, simplified approach to characterising this impedance using a PA with a simple multi-tone modulation as a load. It describes the measurement setup and verifies the results by characterising the baseband impedance of a state-of-the-art buck converter and comparing this to its static model. The results demonstrate that multi-tone signals and complex modulations yield comparable results and are both suitable for measuring and modelling the baseband impedance. This shows that using simple multi-tone measurements and basic equipment, the full static impedance characteristic can be obtained.
\end{abstract}

Index Terms-Power amplifiers, Envelope tracking, HEMT, Broadband, Modulator, Characterisation.

\section{INTRODUCTION}

Modern wireless communication standards require power amplifiers (PAs) that are efficient over a wide bandwidth and a wide power range while maintaining high linearity. The techniques used to achieve efficient operation with signals of high peak-to-average-power-ratios (PAPR) include envelope tracking (ET), Doherty, load-modulated balanced and outphasing PAs [1]-[4]. In ET, the PA is maintained in a high efficiency state by modulating its supply voltage with a dynamic power supply (DPS). This allows the PA to be designed primarily with broad band operation in mind as the efficiency is ensured by the DPS; it does however move the complexity towards the DPS. One critical parameter in ET is the baseband impedance the DPS presents to the PA within the modulation bandwidth, as it has a direct impact on the linearity of the PA [5], [6]. In PAs using constant, static supply voltages, the bias network can be designed to provide a wideband short circuit over the baseband frequency range [6], in ET PAs however, the bias network needs to allow baseband frequencies to pass unhindered, to allow the modulation of the supply voltage at those frequencies. The low impedance at the baseband frequency therefore needs to be provided by the supply modulator itself. Depending on the type of modulator, the baseband impedance is a result of the output stage [7], the output filter [8]-[10] or a combination of both [7]. Knowledge of the baseband impedance is important in ET system design as it informs the PA, system and modulator designers of the distortion that will be introduced by the modulator. This enables judging whether the system work as it is, need additional pre-distortion or a redesign of the modulator. One way of characterising the baseband impedance is presented in literature [7], and uses a PA excited with Schroeder 2Ktone complex modulation, albeit with no verification of the measurements and no presented phase information, preventing the measured data from being used to model the modulator.

In terms of the structure of this paper, section II describes the measurement approach and section III demonstrates measurements on a buck-converter as a case study.

\section{Measurement Setup}

This technique follows the approach presented in [7] and exploits the fact that a current drawn will reveal the impedance by inducing a voltage drop. A constant voltage is supplied by the DUT, here the supply modulator, to a load, resulting in a current flow. If the load draws a time varying current, it will generate a time varying voltage drop over the baseband impedance presented by the modulator which can be measured. In the case of the modulator for an ET system, the obvious time varying load to use is the power amplifier it is supplying, as the current of a PA is easily varied. Importantly, the baseband impedance presented by the bias network of the PA needs to be suitable for this measurement, i.e. terminating only the RF and presenting a through at the baseband frequencies. The schematic in Fig. 1(a) shows that for this measurement, any element to the right of the voltage probe has no impact on the ratio of voltage and current. It will however reduce the magnitude of the current and thus the voltage generated and, with it, the sensitivity of the measurement. The sensitivity of the measurement also depends on the sensitivity of 


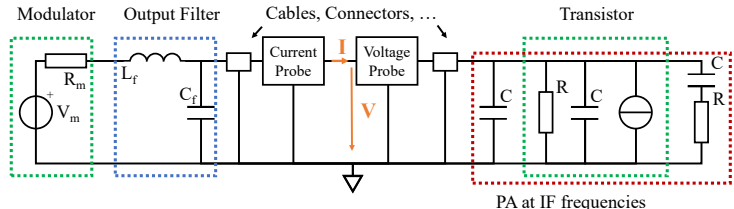

(a)

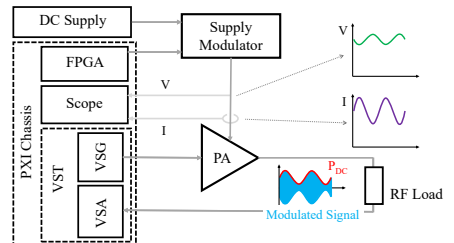

(b)

Fig. 1. (a) Equivalent circuit of the measurement setup and (b) Schematic of the measurement setup.

the oscilloscope and the frequency responses of voltage and current probe. This technique allows the characterisation of the behaviour of baseband impedance over a wide frequency range. To achieve this, the current drawn by the PA needs to cover the frequency range of interest. There are a number of approaches that allow this, for example, the PA can be operated with a chirped amplitude modulation (AM), where the modulation frequency is swept over the required bandwidth, with the desired frequency resolution. Alternatively, the simple chirped AM can be replaced with a communications signal such as WCDMA or LTE, allowing the baseband impedance to be measured over the bandwidth of that signal. Another approach was presented in [7], using an amplitude and phase modulated Schroeder 2K-tone modulation. A third option is using multitone signals to modulate the PA. This has the advantage that it is easy to generate using widely available equipment, it additionally scales well as the modulation frequency can be easily adapted to cover a specific frequency range.

\section{MeAsurements}

To test how the different modulation standards compare in terms of measurement accuracy, a bespoke supply modulator for envelope tracking applications is characterised. This is a state-of-the-art buck-converter with a switching frequency of $75 \mathrm{MHz}$, and an output filter designed to accommodate a modulation bandwidth of $2 \mathrm{MHz}$. The modulator is controlled by a FPGA located in a NI PXI chassis, as shown in Fig. 1(b). Using a $400 \mathrm{MHz} 14$ bit oscilloscope, a voltage probe, and a current probe with bandwidths exceeding $100 \mathrm{MHz}$, the voltage and the current are measured between the PA and modulator. A $2.9 \mathrm{GHz} \mathrm{PA}$ biased in class $\mathrm{AB}$ with a quiescent current of $200 \mathrm{~mA}$ is excited with four different modulated signals; a 2-tone with $1 \mathrm{MHz}$ separation, a five-tone with $1 \mathrm{MHz}$ separation between tones, and two pre-configured communication signals to cover different modulation bandwidths, a $20 \mathrm{MHz}$ LTE signal and a $40 \mathrm{MHz}$ WCDMA signal. The measurement results in Fig. 2(a) demonstrate the time varying current drawn by the PA in response to the two-tone signal. The measured voltage, constant at $15 \mathrm{~V}$ when the PA is not

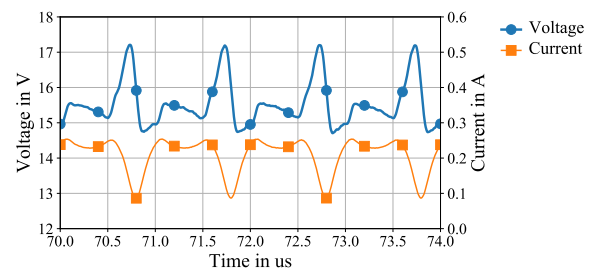

(a)

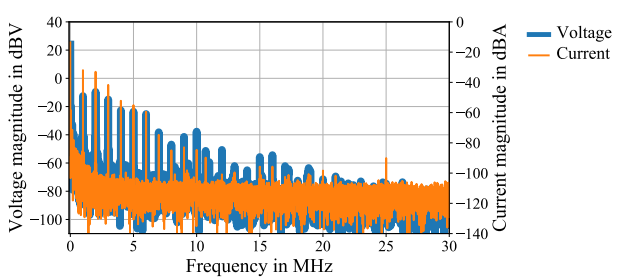

(b)

Fig. 2. Measured voltage and current between PA and modulator with a supply voltage of $15 \mathrm{~V}$ and a two tone excitation (a) in the time domain and (b) in the frequency domain

excited, now also varies in response to the modulation. Interestingly, the voltage and current waveforms look differently, which shows that the magnitude ratio and the phase shift between voltage and current change with frequency. Fig. 2(b) shows that the voltage to current ratio changes significantly with frequency and that the harmonic frequency components stay significantly above the noise floor for frequencies much higher than the modulation frequency of $1 \mathrm{MHz}$. The measured voltage becomes too small to measure above $17 \mathrm{MHz}$ while the current components are measurable up to $10 \mathrm{MHz}$, resulting in an overall characterisation bandwidth of 10 times the modulation frequency. By dividing voltage by current in the frequency domain, the baseband impedance in the frequency domain can be obtained at the modulation frequency and its harmonics, as shown in Fig. 3(a). The magnitude of the baseband impedance can be seen to increase with frequency, as expected for the output filter of the modulator. As the voltage and current spectra in Fig. 2(b) get close to the noise floor as the frequency passes $10 \mathrm{MHz}$, the magnitude and phase of the baseband impedance become erratic as even if the signal stays $20 \mathrm{~dB}$ above the noise floor, the voltage or current measurement can still end up with a random error of up to $10 \%$. The same measurement and analysis procedure is repeated for the other types of modulation, resulting in the baseband impedance in Fig. 3(a). The five-tone modulation occupies a bandwidth of $4 \mathrm{MHz}$ and allows characterisation up to around $24 \mathrm{MHz}$, above which the phase measurements become erratic. The realistic modulations, LTE and WCDMA, use the full spectrum instead of discrete tones, resulting in a continuous baseband impedance measurement result up to around $40 \mathrm{MHz}$, despite their difference in modulation frequency, see Fig. 3(a). The measurements show that the baseband impedances acquired using the different types of modulation show good agreement, with the aforementioned limitations in frequency. This shows that it is unnecessary to use complex or specially crafted modulation signals to characterise baseband impedances.

As verification of the characterisation, the measurement 

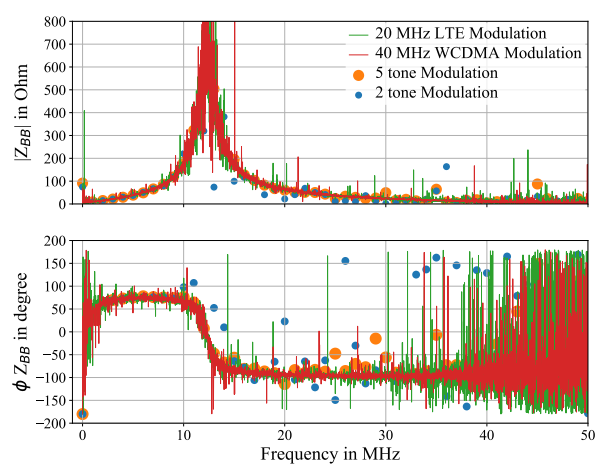

(a)
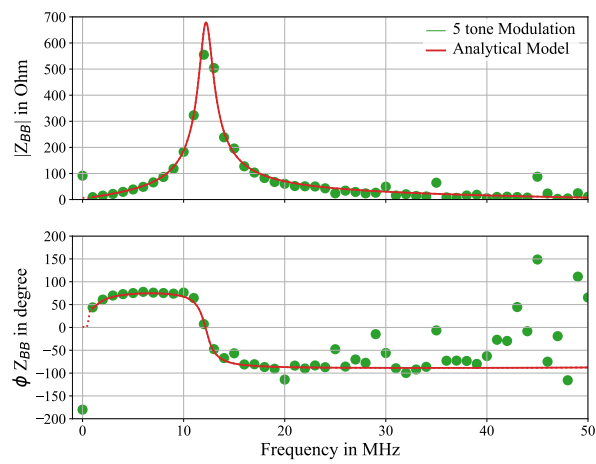

(b)

Fig. 3. Measured baseband impedance at $15 \mathrm{~V}$ using different types of modulation and (b) comparison of modelled (red, solid line, dotted for extrapolated values) and measured (green circles) baseband impedance

results are compared with an equivalent-circuit model of the same modulator, comprising the modulator itself, represented by an average value voltage source $V_{m}=15 \mathrm{~V}$ and a resistor $R_{m}=5.7 \Omega$, and the output filter with a Coilcraft 1812PS102 inductor and a capacitor array consisting of one $120 \mathrm{pF}$ KEMET C0603C121J5G and two $22 \mathrm{pF}$ KEMET C0603C220J5G, as shown in Fig. 1(a), using manufacturer provided s-parameters for the filter components. Fig. 3(b) compares the results of the 5-tone measurement with the model of the baseband impedance, showing very good agreement. Using a buck-converter as a DUT for this case study has the advantage of providing a relatively simple way to verify measurements as its static impedance can be modelled using a simple circuit. Once the output voltage of a buck converter changes however, so does its baseband impedance. Since the filter response stays constant, this change can be attributed to the real part of the baseband impedance, as shown in Fig. 4. These static measurements can provide the data necessary to model the converter, possibly even more relevant in other converter types that are harder to simulate and model, e.g. in hybrid modulators that comprise interacting buck-converters and linear amplifiers [7].

\section{CONCLUSiON}

This paper discussed a way of measuring the baseband impedance of a supply modulator using a PA and a modulated signal. A realisation of the approach has been used to measure

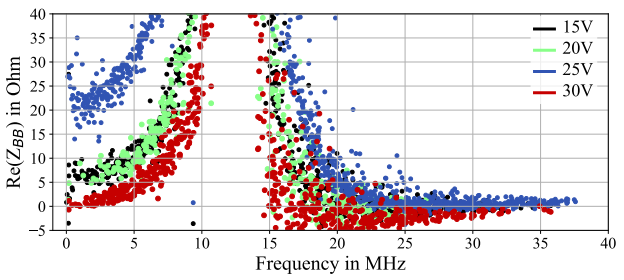

Fig. 4. Measured real part of the baseband impedance at different supply voltages using a WCDMA signal

a buck-converter and has shown that different types of modulation, lead to comparable results and were able to measure the baseband impedance in a single measurement. Both approaches yield the data necessary to evaluate and model static magnitude and phase of the complex baseband impedance of a modulator, showing good agreement with the static model. The measurement also shows how the baseband impedance depends on the output voltage and emphasizes the value of such comprehensive, multi-dimensional measurements, covering a wide frequency and output voltage range. Another important result of this paper is that these measurements can be conducted with complex measurement systems as well as with simple signal generators, with comparable results.

\section{ACKNOWLEDGMENT}

This work was supported by the Engineering and Physical Science Research Council (EPSRC) in the context of the research project EP/N016408/1.

\section{REFERENCES}

[1] Z. Popović, "Amping Up the PA for 5G: Efficient GaN Power Amplifiers with Dynamic Supplies," IEEE Microwave, vol. 18, no. 3, pp. 137-149, May 2017.

[2] R. Quaglia, M. D. Greene, M. J. Poulton, and S. C. Cripps, "Design and characterization of a $1.7-2.7 \mathrm{GHz}$ quasi-MMIC Doherty power amplifier," in IEEE MTT-S Int. Microw. Symp. Dig., Jun. 2017, pp. 771-773.

[3] D. Collins, R. Quaglia, J. Powell, and S. Cripps, "Experimental Characterization of a Load Modulated Balanced Amplifier with Simplified Input Power Splitter," in Proc. Asia-Pacific Microw. Conf., Oct. 2018, pp. 461-463.

[4] T. Cappello, P. H. Pednekar, C. Florian, Z. Popovic, and T. W. Barton, "Supply Modulation of a Broadband Load Modulated Balanced Amplifier," in IEEE MTT-S Int. Microw. Symp. Dig., Jun. 2018, pp. 304-307.

[5] M. Akmal et al., "The effect of baseband impedance termination on the linearity of GaN HEMTs," in Proc. Eur. Microw. Conf., Sep. 2010 , pp. 1046-1049.

[6] L. C. Nunes, D. R. Barros, P. M. Cabral, and J. C. Pedro, "Efficiency Degradation Analysis in Wideband Power Amplifiers," IEEE Trans. Microw. Theory Techn., vol. 66, no. 12, pp. 5640-5651, Dec. 2018.

[7] G. P. Gibiino, J. Couvidat, G. Avolio, D. Schreurs, and A. Santarelli, "Supply-terminal $40 \mathrm{MHz}$ BW characterization of impedance-like nonlinear functions for envelope tracking PAs," in ARFTG Conf. Dig., May 2016, pp. 1-4.

[8] A. Villarruel-Parra and A. Forsyth, "75 MHz discrete GaN based multi-level buck converter for envelope tracking applications," in IEEE Applied Power Electronics Conference, Mar. 2019, pp. 1553 1560.

[9] S. Paul et al., "Analysis of Dissipated Power in Envelope Amplifier Output Filters," in Proc. Eur. Microw. Conf., Sep. 2018, pp. 515-518.

[10] J. A. Garcia, R. Marante, M. N. Ruiz, and G. Hernandez, "A 1 $\mathrm{GHz}$ frequency-controlled class $E^{2}$ DC/DC converter for efficiently handling wideband signal envelopes," in IEEE MTT-S Int. Microw. Symp. Dig., Jun. 2013, pp. 1-4. 